\title{
On Small Subsets in Euclidean Spaces
}

by

\author{
Jan MYCIELSKI and Grzegorz TOMKOWICZ \\ Presented by Stanisław KWAPIEŃ
}

\begin{abstract}
Summary. We study a property of smallness of sets which is stronger than the possibility of packing the set into arbitrarily small balls (i.e., being Tarski null) but weaker than paradoxical decomposability (i.e., being a disjoint union of two sets equivalent by finite decomposition to the whole). We show, using the Axiom of Choice for uncountable families, that there are Tarski null sets which are not small sets. Using only the Principle of Dependent Choices, we show that bounded subsets of $\mathbb{R}^{n}$ that are included in countable unions of proper analytic subsets of $\mathbb{R}^{n}$ are small, and several related results.
\end{abstract}

1. Introduction. Two metric spaces $X$ and $Y$ are equivalent by finite decomposition (denoted by $X \equiv Y$ ) if there exist finite partitions $X_{1}, \ldots, X_{n}$ and $Y_{1}, \ldots, Y_{n}$ of $X$ and $Y$, respectively, such that $X_{k}$ is congruent to $Y_{k}$ for $k=1, \ldots, n$. We say that the space $X$ can be packed into a space $Y$ if there exists a set $X^{\prime} \subseteq Y$ such that $X^{\prime} \equiv X$. We say that a set $S \subseteq X$ is Tarski null if it can be packed into any ball $K \subseteq X$ of positive radius. The sets with this property were studied by Tarski [11], [12] and later by Hadwiger [3]. We say that $K$ absorbs $S$ if there exists an $S^{\prime} \subseteq K$ such that $S^{\prime} \equiv S$ and $K \backslash S^{\prime} \equiv K$.

In the present paper we introduce a new notion of smallness. We say that $S$ is small if every ball of positive radius absorbs $S$. Thus every Tarski null set is bounded and every small set is Tarski null. Of course, by our definitions, if $X$ is dense in itself then Tarski null sets and small sets form invariant ideals and all these sets are bounded.

2010 Mathematics Subject Classification: Primary 03E05, 20E05, 51M05; Secondary $20 \mathrm{G} 20$.

Key words and phrases: analytic set, equivalence by finite decomposition, small set, Tarski absolute measure.

Received 30 September 2016.

Published online 21 October 2016. 
We will also relativize the above notions to subgroups $G$ of groups of isometries which acts transitively on $X$, i.e., $\equiv_{G}$ will denote equivalence which uses only isometries of $G$.

A set $E \subseteq X$ is $G$-paradoxical if $E$ can be partitioned into two sets $A$ and $B$ such that $E \equiv_{G} A \equiv_{G} B$. We will omit the prefix or subscript $G$ when the intended $G$ is clear from the context. We recall that there are bounded paradoxical sets in $\mathbb{R}^{2}$, even ones including perfect sets (i.e., non-empty, closed and without isolated points), and this does not require the Axiom of Choice (see [13, Theorem 14.15]), but there are no paradoxical sets in $\mathbb{R}([13$, Cor. 14.25]).

We will show that every bounded $G$-paradoxical subset of the Euclidean space $\mathbb{R}^{n}$ is also a small set (Theorem 5.1).

We will show that there are perfect sets in the real line $\mathbb{R}$ which are small (Corollary 3.3), and that there are Tarski null sets in $\mathbb{R}$ which are not small (Corollary 3.8). We do not know if there are such sets in $\mathbb{R}^{n}$ for $n>1$.

The following simple problem is still unsolved: is the classical Cantor set small in $\mathbb{R}$ ?

Let $\mathbb{S}^{n}$ and $\mathbb{H}^{n}$ denote the $n$-dimensional sphere and hyperbolic space, respectively. By theorems of Banach and Tarski [1] and ours [5, 6] every bounded set in $\mathbb{R}^{n}$ for $n>2$, and in $\mathbb{S}^{n}$ and in $\mathbb{H}^{n}$ for $n>1$ is small. Of course, this theorem depends on the Axiom of Choice for uncountable families of sets of real numbers $\left(\mathrm{AC}_{\mathbb{R}}\right)$. But the concept of a small set in these spaces does not trivialize if we forbid $\mathrm{AC}_{\mathbb{R}}$ and accept only the Principle of Dependent Choices (DC); for related investigations see [7]. First, by a proper analytic subset of a connected analytic manifold we mean the set of zeros of some non-constant analytic function. We will show, using only DC, that all bounded sets in $\mathbb{R}^{n}, \mathbb{S}^{n}$ and $\mathbb{H}^{n}$ that are subsets of countable unions of proper analytic subsets are small (Theorem 4.1). We will also show that a finite union of convex closed curves (i.e. boundaries of bounded convex sets) in $\mathbb{R}^{2}$ is small. This should generalize to $\mathbb{R}^{n}$, but we have no proof. We conjecture that every bounded set which is a union of countably many convex surfaces in $\mathbb{R}^{n}$ (that is, boundaries of bounded convex sets) is small.

In [11] Tarski proved certain measure-theoretic theorems in $\mathbb{R}$ and mentioned the possibility of extending them to $\mathbb{R}^{2}$. But this extension is not routine. One of his main results is a characterization of his nullsets in $\mathbb{R}$ [11, Satz 2.20]. Following his suggestion we will extend this result to $\mathbb{R}^{n}, \mathbb{S}^{n}$ and $\mathbb{H}^{n}$ (Theorem 5.2).

2. Generalities. First we summarize Tarski's theory of semigroups of types and their measures. 
DeFinition 2.1. Let a group $G$ act transitively on an unbounded metric space $X$ and let $\mathcal{B}$ be the Boolean ring of all bounded subsets in $X$. Define the type $[A]$ of a set $A \in \mathcal{B}$ as $\left\{B \in \mathcal{B}: A \equiv_{G} B\right\}$. Now we define the semigroup of types, $\mathcal{S}(X, G)$, in the following way: $[A]+[B]=[A \cup \tau(B)]$, where $\tau$ is an element of $G$ such that $A \cap \tau(B)=\emptyset$. Note that + is associative, commutative and has a neutral element $0=[\emptyset]$.

$n \cdot \alpha:=\alpha+\cdots+\alpha$ with $n$ summands. Also, we introduce an order in $\mathcal{S}$, saying that $[A] \leq[B]$ if there exists $A^{\prime} \subseteq B$ such that $A^{\prime} \equiv A$.

$\mu: \mathcal{S}(X, G) \rightarrow \mathbb{R}$ is called a measure on $\mathcal{S}(X, G)$ if for all $\alpha, \beta \in \mathcal{S}(X, G)$, $\mu(\alpha) \geq 0$ and $\mu(\alpha+\beta)=\mu(\alpha)+\mu(\beta)$.

THEOREM 2.2 .

(i) If $n \cdot \alpha \geq(n+1) \cdot \alpha$, then $2 \cdot \alpha=\alpha$ (Tarski; [13, Cor. 10.21]),

(ii) If ( $\alpha \leq \beta$ and $\beta \leq \alpha$ ), then $\alpha=\beta$ (Banach-Schröder-Bernstein; [13, Thm. 3.6]),

(iii) If $n \cdot \alpha \leq n \cdot \beta$, then $\alpha \leq \beta$ (König and Valko; [13, Thm. 10.20]).

The following is the main theorem of Tarski's theory [13, Thm. 11.1]:

THEOREM 2.3. $2 \cdot \varepsilon \neq \varepsilon$ if and only if there exists a measure $\mu$ on $\mathcal{S}(X, G)$ such that $\mu(\varepsilon)=1$.

Now we turn to some properties of the relation $\equiv$, pertaining to the smallness and absorbability defined at the begining of Section 1.

Proposition 2.4. Let $A \equiv A^{\prime}$ and $B \equiv B^{\prime}$. Then:

(i) $A$ absorbs $B$ [can be packed in $B]$ if and only if $A^{\prime}$ absorbs $B^{\prime}[$ can be packed in $\left.B^{\prime}\right]$.

(ii) If $A \cap B=\emptyset=A^{\prime} \cap B^{\prime}$, then $A \cup B \equiv A^{\prime} \cup B^{\prime}$.

Proof. The only nontrivial part pertains to absorbability: $A \equiv A^{\prime}$ is witnessed by a bijection $f: A \rightarrow A^{\prime}$. Assuming that $A$ absorbs $B$ there exists $C \subseteq A$ such that $C \equiv B$ and $A \backslash C \equiv A$. We find that $A \backslash C \equiv A^{\prime} \backslash f(C)$, and since $A \backslash C \equiv A \equiv A^{\prime}$, the transitivity of $\equiv$ implies that $A^{\prime} \equiv A^{\prime} \backslash f(C)$ and $f(C) \equiv B^{\prime}$. So $A^{\prime}$ absorbs $B^{\prime}$.

Proposition 2.5. Let $G$ be the group of isometries acting on a metric space $X$. Then the family of $G$-small sets is a $G$-invariant ideal.

Proof. It is obvious that every subset of a small set is small. Let $K$ be a ball of positive radius. Then since $K$ absorbs $A, K$ is infinite. Thus $K$ contains disjoint balls $K_{1}$ and $K_{2}$ such that $A \equiv A_{1} \subseteq K_{1}, K_{1} \equiv K_{1} \backslash A_{1}$ and $B \equiv B_{1} \subseteq K_{2}, K_{2} \equiv K_{2} \backslash B_{1}$. This implies $A \cup B \equiv A_{1} \cup B_{1}$ and $K \equiv K \backslash\left(A_{1} \cup B_{1}\right)$. 
3. Small sets in $\mathbb{R}, \mathbb{S}$ and Vitali sets. In this section we denote by $\mathbb{S}$ any circle or its group of rotations, by $\mathbb{R}$ a straight line or its group of translations, and by $\mathbb{Q}$ the set or additive group of rational numbers. We use the arc-length metric defined on $\mathbb{S}$ such that the distance between $x$ and $y$ is the length of the shortest arcs from $x$ to $y$ in $\mathbb{S}$, and the usual distance in $\mathbb{R}$. We could use any distance invariant under the group operations consistent with the standard topologies. The diameter of $\mathbb{R}$ could be finite, but a subset of $\mathbb{R}$ will be called bounded if its closure is compact. In this section we will consider mostly the relations $\equiv_{\mathbb{S}}$ and $\equiv_{\mathbb{R}}$, and omit the subscripts.

LEMMA 3.1.

(i) In $\mathbb{R}^{n}, A \equiv B$ implies $s(A) \equiv s(B)$ for any similarity $s$.

(ii) If $A, B \subset \mathbb{S}, A \equiv B$ and $f(x)=e^{i 2 \pi x}$ for $x \in[0,1)$, then $f^{-1}(A) \equiv$ $f^{-1}(B)$.

Proof. Obvious.

With the use of Proposition 2.4 the theorems of Sierpiński [10, Theorem 16] and Tarski [11, Satz 1.20] generalize as follows.

THEOREM 3.2.

(i) If $W$ is a subgroup of $\mathbb{S}$ and there exists an infinite cyclic group $C \subset \mathbb{S}$ such that $W \cap C=\{1\}$, then $W$ is small.

(ii) If $W$ is a bounded subset of a subgroup $G$ of $\mathbb{R}$ and $G \cap H=\{0\}$, where $H$ is a subgroup dense in $\mathbb{R}$, then $W$ is small.

Proof. (i) Let $I \subset \mathbb{S}$ be an arc of length $l$. We split $G$ into sets of diameter less than $l$. Then using rotations of the form $\rho^{m}$, where $\rho \in \mathbb{S} \backslash W$ is an absorbing element, we pack those sets into $I$. Now we take a circle $\mathbb{S}^{\prime}$ of length $l$ with the arc-length metric and let $G^{\prime} \subset \mathbb{S}^{\prime}$ be such that $G \equiv G^{\prime}$. It is clear that $G^{\prime}$ is absorbable in $\mathbb{S}^{\prime}$ using the same $\rho$ acting on $\mathbb{S}^{\prime}$. Hence, by Proposition 2.4, $G$ is a small subset of $\mathbb{S}$.

(ii) We may assume that $H$ is countable. The set $\mathbb{Q}$ of rationals is countable, and therefore there are countably many similarities $s$ such that $s(H) \cap \mathbb{Q} \neq \emptyset$. So we can pick a similarity $s$ such that $s(H) \subset[0,1)$ and $s(H) \cap \mathbb{Q}=\emptyset$. Now use (i) and Lemma 3.1.

Corollary 3.3.

(i) Every bounded set $C$ of $\mathbb{R}$ or of $\mathbb{S}$ of cardinality less than $2^{\aleph_{0}}$ is small.

(ii) There exist perfect sets in $\mathbb{R}$ and $\mathbb{S}$ that are small, and such sets can be constructed without using the Axiom of Choice.

Proof. (i) This follows from Theorem 3.2(ii). 
(ii) As is well known, there are perfect sets $P$ that are linearly independent over $\mathbb{Q}(\pi)$ and contained in $[0,1)$ (see $\left[4\right.$, Theorem 1]). Moreover, $e^{i 2 \pi x}$ restricted to the open interval $(0,1)$ is a homeomorphism. Thus, applying the rotation through 1 radian, by Lemma 3.1 and Theorem 3.2 we see that $P$ is small.

We will show that there are sets in $\mathbb{S}$ and $\mathbb{R}$ which are Tarski null but are not small.

A set will be called a Vitali set if it is a choice set from the cosets of the factor group $\mathbb{S} / T$, where $T$ is the subgroup of elements of finite order in $\mathbb{S}$. By the structure theorem for abelian divisible groups, $\mathbb{S}$ is isomorphic to the direct sum $T \oplus \mathbb{S} / T$ and $\mathbb{S} / T$ is of the form $\bigoplus_{i \in I} \mathbb{Q}$, where $|I|=2^{\aleph_{0}}$.

Proposition 3.4. Every Vitali set $V \subset \mathbb{S}$ and every proper subgroup $G$ of $\mathbb{S}$ is Tarski null.

Proof. The group generated by a Vitali set is a proper subgroup of $\mathbb{S}$. So it suffices to show the second part of the proposition. Take an $\operatorname{arc} L \subset \mathbb{S}$ of length $d$ and divide $\mathbb{S}$ into finitely many $\operatorname{arcs} L_{1}, \ldots, L_{k}$ of length less than $d$. Of course $\mathbb{S} \backslash G$ is dense in $\mathbb{S}$. So let $G_{i}=G \cap L_{i}$ for $i=1, \ldots, k$ and choose some $\rho_{1}, \ldots, \rho_{k} \in \mathbb{S} \backslash G$ such that $\rho_{i}\left(G_{i}\right) \subset L$ for $i=1, \ldots, k$. Then $\rho_{i}\left(G_{i}\right) \cap \rho_{j}\left(G_{j}\right)=\emptyset$ for $i \neq j$. Therefore $G$ can be packed in $L$.

The following theorem is well known (so we omit its proof).

THEOREM 3.5.

(i) Any maximal subgroup $G$ of $\mathbb{S}$ such that $G \cap T=\{1\}$ is a Vitali set, i.e. a selector from $\mathbb{S} / T$.

(ii) There are $2^{2^{\aleph_{0}}}$ such subgroups $G$.

(iii) $\mathbb{S}=T \oplus G$.

In contrast to Theorem 3.2(i) and Proposition 3.4 we have the following:

THEOREM 3.6. If $V$ is a Vitali set which is a subgroup of $\mathbb{S}$, then $\mathbb{S} \equiv$ $\mathbb{S} \backslash V$ fails (whence $V$ is Tarski null but not small).

In order to prove this theorem recall that a group $G$ is called supramenable if for any nonempty $A \subseteq G$ there is a finitely additive, left-invariant universal measure normalizing $A$, and all abelian groups are supramenable (see [13, Theorem 14.4]). It is clear that if $G / H$ is finite and $H$ is supramenable then $G$ is supramenable. Hence the group of all isometries of $\mathbb{S}$ is supramenable.

To prove Theorem 3.6 we need the following lemma:

LEMma 3.7. If $G$ is a subgroup of $\mathbb{S}$ which is a Vitali set, then every isometric image of $G$ in $\mathbb{S}$ is of the form $t G$, where $t \in T$.

Proof. We begin with two remarks: every isometry between two subsets of $\mathbb{S}$ extends to an isometry of $\mathbb{S}$; each isometry of $\mathbb{S}$ is of the form $x \mapsto r x$ 
or $x \mapsto r x^{-1}$, where $r$ is a rotation. By Theorem 3.5(iii), $r$ is of the form $t g$, where $t \in T$ and $g \in G$. Therefore

$$
r G=\operatorname{tg} G=t G \quad \text { and } \quad r G^{-1}=r G=\operatorname{tg} G=t G .
$$

Proof of Theorem 3.6. By Proposition 3.4, $V$ is Tarski null. So we must show that $V$ is not absorbable.

Assume to the contrary that $V$ is absorbable, i.e., there is a bijection $f: \mathbb{S} \rightarrow \mathbb{S} \backslash V$ such that $f=f_{1} \cup \cdots \cup f_{k}$, where each $f_{i}$ is the restriction of a map $r_{i} x=t_{i} v_{i} x$ or $r_{i} x^{-1}=t_{i} v_{i} x^{-1}$, where $t_{i} \in T$ and $v_{i} \in V$. Hence, $f^{p}(V) \cap f^{q}(V)=\emptyset$ for all distinct nonnegative integers $p$ and $q$. Let $H$ be the subgroup of $T$ generated by $t_{1}, \ldots, t_{k}$. Being a finitely generated subgroup of $T, H$ is finite. Let us prove the following

Claim. $f(H V) \subseteq H V$.

Proof. Let $h v \in H V$, where $h \in H$ and $v \in V$. Then for some $i \leq k$,

$$
f(h v)=f_{i}(h v)=t_{i} h v \quad \text { or } \quad f(h v)=t_{i}(h v)^{-1} .
$$

Hence

$$
f(h v)=h^{\prime} v \quad \text { or } \quad f(h v)=h^{\prime} v^{-1},
$$

where $h^{\prime} \in H$. Thus, since $V$ is a group, in both cases $f(h v) \in H V$, which proves the Claim.

Now we will apply the semigroup of types $\mathcal{S}(\mathbb{S}, G)$, where $G$ is the group of all isometries of $\mathbb{S}$. Let $m$ be the order of $H$. Thus $[H V]=m[V]$. By the Claim, since $f: \mathbb{S} \rightarrow \mathbb{S} \backslash V$, we have $f: H V \rightarrow H V \backslash V$. Hence $[H V] \leq$ $[H V \backslash V]$, and

$$
m[V]=[H V] \leq[H V \backslash V]=(m-1)[V] .
$$

Thus, by Theorem 2.2, we obtain $2[V]=[V]$. But this is impossible since $G$ is supramenable.

COROLlary 3.8. There is a subset of $\mathbb{R}$ which is a Tarski null set but is not a small set.

Proof. By Proposition 2.4 and Theorem 3.6.

We have two unsolved problems related to Theorem 3.6:

Problem. 1. Does there exist a Vitali set $V$ which is a small set in $\mathbb{S}$ ?

2. Does there exist a measurable set or a set with the property of Baire which is Tarski null but not small?

4. Small sets in higher dimensions. Let $\mathbb{X}^{n}$ be one of the spaces: $\mathbb{R}^{n}, \mathbb{S}^{n}$ or $\mathbb{H}^{n}$, where $n>1$. Let $\Sigma$ be an o-minimal structure on the field $\mathbb{R}$. We will consider subsets of $\mathbb{X}^{n}$ which are definable (with parameters) in $\Sigma$. The most natural examples of such definable sets are subanalytic sets, i.e., 
sets definable in the expansion of $\mathbb{R}$ by analytic functions whose domains are restricted to compact subsets of $\mathbb{X}^{n}$, and quasi-subanalytic sets, i.e., sets definable in the expansions of $\mathbb{R}$ by restricted quasi-analytic functions (with respect to various quasi-analytic classes).

For $\mathbb{R}^{n}, n \geq 3$, and $\mathbb{S}^{n}$ or $\mathbb{H}^{n}, n \geq 2$, the next theorem follows immediately from Banach-Tarski paradoxical decompositions (see [13]), but of course this requires the Axiom of Choice for uncountable families of sets (AC). But our next theorem will be proved using only DC. We will apply a theorem proved in 9 .

THEOREM 4.1. Let $A$ be a bounded set in $\mathbb{X}^{n}$ such that $A \subseteq \bigcup_{i=1}^{\infty} A_{i}$, where $A_{i}$ are subsets of $\mathbb{X}^{n}$ definable in $\Sigma$, and $\operatorname{dim} A_{i}<n$ for all $i$. Then $A$ is small.

Proof. For any set $Y \subseteq \mathbb{X}^{n}$ we define

$$
d(Y)=\min \left\{\max \left\{\operatorname{dim} A_{i}: i=1,2, \ldots\right\}: Y \subseteq \bigcup_{i=1}^{\infty} A_{i}\right. \text { and }
$$

$A_{i}$ are definable in $\left.\Sigma\right\}$.

$d(Y)$ will be called the $\Sigma$-dimension of $Y$. We observe that if $Y=Y_{1} \cup Y_{2} \cup \cdots$, then $d(Y)=\max \left\{d\left(Y_{i}\right): i=1,2, \ldots\right\} \leq n$.

Now we will argue by induction on $d(A)$. For $d(A)=-1$ the theorem is obvious since then $A=\emptyset$. Suppose the theorem holds whenever $d(A)<$ $d<n$. We have to show that for $\operatorname{dim} A=d, A$ is absorbable by every ball $B \subset \mathbb{X}^{n}$ of positive radius.

Let $\mathfrak{R}_{n}$ be the set of all rotations of $\mathbb{X}^{n}$ whose set of fixed points is a hyperplane of dimension $n-2$ (of course by a hyperplane in $\mathbb{S}^{n}$ we mean a large sphere).

We choose a closed ball $K$ in the interior of $B$ which does not contain the center of $B$.

Now we split $A$ into finitely many sets of diameters less than the radius of $K$. By the theorem of [9] there exist rotations in $\Re_{n}$ which move those sets into $K$ in such a way that the $\Sigma$-dimension of the intersection of the images of any two of them is less than $d$. Hence the union $U$ of the inverse images of these intersections is also of $\Sigma$-dimension less than $d$. So by the inductive assumption $U$ is absorbable in $B$. Thus it suffices to show that $A \backslash U$ is also absorbable in $B$.

By our choice of $K$, it is clear that if the set of fixed points of $\rho \in \mathfrak{R}_{n}$ is sufficiently close to the center of $B$ then $\bigcup_{n=0}^{\infty} \rho^{n}(K) \subseteq B$. Thus we have a nonempty open set $O \subseteq \mathfrak{R}_{n}$ such that for all $\rho \in O, \bigcup_{n=0}^{\infty} \rho^{n}(K) \subseteq B$.

Using again the theorem of [9] we find a $\rho \in O$ such that the $\Sigma$-dimensions of all intersections of the set $\rho^{n}(A \backslash U)$ are smaller than $d$. So the union $V$ of 
the inverse images of these intersections is also of $\Sigma$-dimension less than $d$, and hence absorbable by $B$. It remains to show that $(A \backslash U) \backslash V$ is absorbable by $B$-but this is obvious since any two sets $\rho^{n}((A \backslash U) \backslash V)$ are pairwise disjoint.

Theorem 4.1 should also be true if the $A_{i}$ are convex surfaces in $\mathbb{X}^{n}$, i.e., boundaries of bounded convex sets. Unfortunately, in general the latter are not definable in o-minimal structures. We have been able to prove only the following case of the conjecture.

THEOREM 4.2. Every bounded set $A \subset \mathbb{R}^{2}$ which is a finite union of convex curves is small.

Proof (outline). Choose any disk $D$ with center $c$. Let $C$ be a convex curve. Hence $C$ has at most four points whose interior angles are less than or equal to $\pi / 4$. Hence $C$ can be covered by finitely many $\operatorname{arcs}(a, b]$ such that $(a, b)$ does not contain any vertex with interior angle smaller than $\pi / 4$ and it has a translation $t$ which moves it into $D$ such that $t(a)=c$. Then every circle in $D$ concentric with $D$ intersects $t((a, b])$ exactly once. Thus an appropriate rotation of $D$ shows that $D$ absorbs $(a, b]$. Then by Proposition 2.5, $C$ is small, and Theorem 4.2 follows.

Problem. 3. Is every bounded convex surface in $\mathbb{R}^{n}$ small?

\section{Bounded paradoxical sets are small and Tarski null sets are al-} most absorbable. Recall that $\mathbb{X}^{n}$ denotes $\mathbb{R}^{n}, \mathbb{S}^{n}$ or $\mathbb{H}^{n}$, where $n=1,2, \ldots$ Recall also that even in $\mathbb{X}^{2}$ there are bounded paradoxical sets (with some additional properties such as having perfect subsets and being of positive outer measure; see [2] and [13, Section 14.2]).

The following two theorems are based on a common lemma,

TheOREM 5.1. Let $G$ be a subgroup of isometries of $\mathbb{X}^{n}$ (possibly amenable) which acts transitively on $\mathbb{X}^{n}$. Then every bounded $G$-paradoxical subset of $\mathbb{X}^{n}$ is G-small.

The next theorem generalizes Tarski's [11, Satz 2.20].

Theorem 5.2. Let $G$ be as in Theorem 5.1. Then for every $A \subset \mathbb{X}^{n}$ the following are equivalent:

(i) A is G-Tarski null,

(ii) there exists an infinite sequence $A_{1}, A_{2}, \ldots$ of pairwise disjoint sets such that $A_{1} \cup A_{2} \cup \cdots$ is bounded and $A \equiv_{G} A_{1} \equiv_{G} A_{2} \equiv_{G} \cdots$.

Observe that condition (ii) is similar to, but weaker than, absorbability of $A$ by $A_{1} \cup A_{2} \cup \cdots$.

To prove these theorems we will need the following lemma (which applies to the semigroup of types introduced in Section 2). 
Lemma 5.3. Let $G$ be as above and $\varepsilon \in \mathcal{S}\left(\mathbb{X}^{n}, G\right)$ be the type of a compact subset of $\mathbb{X}^{n}$. If $\alpha \in \mathcal{S}\left(\mathbb{X}^{n}, G\right)$ is such that $m \cdot \alpha \leq k \cdot \varepsilon$ for $m=1,2, \ldots$ and some constant $k>0$, then any representative set of $\alpha$ is G-Tarski null.

Proof. Choose any real $a>0$. For any representative set $E$ of $\varepsilon$ there exists an integer $r$ such that $E$ can be covered by $r$ sets which are $G$-congruent and of diameters less than $a$. Let $\gamma$ be the type of these sets. Then $\varepsilon \leq r \cdot \gamma$ and $k r \cdot \alpha \leq k \cdot \varepsilon \leq k r \cdot \gamma$. Hence by Theorem 2.2(iii) we have $\alpha \leq \gamma$. That is, any representative of $\alpha$ can be packed, using some isometries of $G$, into any ball of positive radius in $\mathbb{X}^{n}$.

Proof of Theorem 5.1. Let $P$ be $G$-paradoxical. Then $m \cdot[P] \leq[P]$ for $m=1,2, \ldots$ Hence Lemma 5.3 implies that $P$ is $G$-Tarski null.

It remains to show that $P$ is absorbable by any ball $K$. Now, $P$ can be partitioned into sets $A$ and $B$ such that $A \equiv_{G} P \equiv_{G} B$. Let $K$ be any ball. Then since $P$ is a $G$-Tarski null, there exists a $P^{\prime} \subset K$ with $P^{\prime} \equiv_{G} P$. Hence $P^{\prime}$ can be partitioned into sets $A^{\prime}$ and $B^{\prime}$ such that $A^{\prime} \equiv_{G} P^{\prime} \equiv_{G} B^{\prime}$. This implies $K \backslash B^{\prime}=\left(K \backslash P^{\prime}\right) \cup A^{\prime} \equiv_{G}\left(K \backslash P^{\prime}\right) \cup P^{\prime}=K$. Thus $B^{\prime}$ is $G$-small, and hence $B$ is $G$-small. In the same way we show that $A$ is $G$-small. Since $P=A \cup B$, by Proposition 2.5, $P$ is $G$-small.

Proof of Theorem 5.2. (i) $\Rightarrow$ (ii). Since in $\mathbb{X}^{n}$ any ball of positive radius includes infinitely many balls of positive radii, this implication readily follows from the definition of $G$-Tarski null sets.

(ii) $\Rightarrow$ (i). Let $\alpha \in \mathcal{S}\left(\mathbb{X}^{n}, G\right)$ be the type of $A$. Since $\bigcup A_{i}$ is bounded and for every positive integer $m$ we have $m \cdot \alpha \leq\left[\bigcup A_{i}\right]$, Lemma 5.3 shows that $A$ is $G$-Tarski null.

The proof of the König and Valko Cancellation Law (Thm. 2.2(iii)) which is used in the proof Lemma 5.3 requires the Axiom of Choice for uncountable families of sets. So we have the following:

Problem 5.4. Assuming large cardinal axioms, are Theorems 5.1 and 5.2 true in the universe $L(\mathbb{R})$ ?

\section{References}

[1] S. Banach et A. Tarski, Sur la décomposition des ensembles de points en parties respectivement congruents, Fund. Math. 6 (1924), 244-277.

[2] M. R. Burke, Paradoxical decompositions of planar sets of positive outer measure, J. Geom. 79 (2004), 56-58.

[3] H. Hadwiger, Absolut messbare Punktmengen im euklidischen Raum, Comment. Math. Helv. 28 (1954), 119-148.

[4] J. Mycielski, Independent sets in topological algebras, Fund. Math. 55 (1964), 139147 . 
[5] J. Mycielski, The Banach-Tarski paradox for the hyperbolic plane, Fund. Math. 132 (1989), 143-149

[6] J. Mycielski and G. Tomkowicz, The Banach-Tarski paradox for the hyperbolic plane (II), Fund. Math. 222 (2013), 289-290.

[7] J. Mycielski and G. Tomkowicz, Shadows of the Axiom Choice in L(R), in preparation.

[8] K. J. Nowak, A theorem on generic intersections in an o-minimal structure, Fund. Math. 227 (2014), 21-25.

[9] K. J. Nowak and G. Tomkowicz, Intersection of generic rotations in some classical spaces, Bull. Polish Acad. Sci. Math. 64 (2016), 105-107.

[10] W. Sierpiński, On the Congruence of Sets and Their Equivalence by Finite Decomposition, Lucknow Univ., 1954; reprinted by Chelsea, 1967.

[11] A. Tarski, Über das absolute Mass linearer Punktmengen, Fund. Math. 30 (1938), $218-234$.

[12] A. Tarski, Algebraische Fassung des Massproblems, Fund. Math. 31 (1938), 47-66.

[13] G. Tomkowicz and S. Wagon, The Banach-Tarski Paradox, 2nd ed., Cambridge Univ. Press, 2016.

Jan Mycielski

Department of Mathematics

University of Colorado

Boulder, CO 80309-0395, U.S.A.

E-mail: jmyciel@euclid.colorado.edu
Grzegorz Tomkowicz

Centrum Edukacji $G^{2}$

Moniuszki 9

41-902 Bytom, Poland

E-mail: gtomko@vp.pl 